TEACHING AND LEARNING ETHICS

\title{
Avoiding evasion: medical ethics education and emotion theory
}

C Leget

J Med Ethics 2004;30:490-493. doi: 10.1136/ime.2003.004697

Beginning with an exemplary case study, this paper diagnoses and analyses some important strategies of evasion and factors of hindrance that are met in the teaching of medical ethics to undergraduate medical students. Some of these inhibitions are inherent to ethical theories; others are connected with the nature of medicine or cultural trends. It is argued that in order to avoid an attitude of evasion in medical ethics teaching, a philosophical theory of emotions is needed that is able to clarify on a conceptual level the ethical importance of emotions. An approach is proposed with the help of the emotion theory Martha Nussbaum works out in her book Upheavals of thought. The paper ends with some practical recommendations.

Correspondence to: C Leget, University Medical Centre Nijmegen, PO Box 9101, 232 EFG Nijmegen, $6500 \mathrm{HB}$, the Netherlands; c.leget@efg.umcn.nl

Received 7 May 2003

In revised form

10 September 2003

Accepted for publication

24 September 2003
$\mathrm{T}$ here are various factors that hamper medical ethics education of undergraduate medical students. In recent literature misconceptions such as skepticism (the belief that ethics has no right or wrong answers) and subjectivism (the belief that ethics is whatever any person feels is right), undermining the very teaching of ethics, have been identified. ${ }^{1}$ Alternative approaches have been proposed like education in virtue ethics or the study of literature as a way of broadening and deepening the inner lives of medical students and encourage moral reflectiveness. $^{2}$ Despite many contributions on the level of the teaching material (literature) and objectives (attitude, virtues), one of the major basic problems in my opinion-the evasion of ethics teaching-seems to be not an issue in the literature.

Beginning with an exemplary case study, this paper diagnoses and analyses some important strategies of evasion and factors of hindrance. Some of these inhibitions are inherent to ethical theories; others are connected with the nature of medicine or cultural trends. Consequently, it is argued that in order to avoid an attitude of evasion in medical ethics teaching, a philosophical theory of emotions is needed that is able to clarify on a conceptual level the ethical importance of emotions. An approach is proposed with the help of the emotion theory Martha Nussbaum works out in her book Upheavals of thought. ${ }^{3}$ The paper ends with some practical recommendations.
CASE STUDY: DEATH ON REQUEST

In their third year, medical students of the University Medical Centre Nijmegen, the Netherlands, are confronted with the 1994 documentary Death on request. ${ }^{4}$ The film shows how a patient, who had been diagnosed as having amyotrophic lateral sclerosis, is being put to death by euthanasia. ${ }^{5}$ In preparation the students have studied an extensive part of a Dutch medical ethics handbook in which the many arguments for and against euthanasia are listed. ${ }^{6}$ The textbook does not take sides in this controversial subject. After the students have watched the one hour video in a group of 15 people, they are invited to share their feelings about the subject, after which they are asked how they judge the role of the physician according to the criteria given by the Dutch law. Finally they are asked whether they think that the arguments against euthanasia are sufficiently taken into account in the video.

As one might expect of a subject as controversial as euthanasia, the video has great impact on the students. All students are emotionally involved in the subject, some even to the extent of leaving the room in tears. The following discussion on the sharing of emotions, however, does not automatically take place on the level of ethics. Four moves are made that contribute to an evasion of ethical discussion. The paradox here, however, is that all four moves are endorsed by popular versions of different ethical theories.

\section{FOUR RESPONSES, FOUR THEORIES}

The first response is made by those who consider euthanasia a legal rather than an ethical matter. Since the Dutch law offers the possibility of euthanasia, the subject at discussion is basically one of the individual doctor and patient as to whether they come to an agreement. This attitude is endorsed by a particular version of liberal individualism. Liberalism, as a tradition in ethical and political thought, has contributed to peaceful coexistence and discussion in a situation of disagreement. The version of liberal individualism embraced by some of the students, however, lacks any awareness of the political dimension and importance of this tradition. This version inhibits ethical discussion by banning all ethical discussion to the personal sphere and subsequently reducing it to a matter of agreement.

A second response is made by students who reason that the fact of whether this individual patient died from euthanasia or from further progression of amyotrophic lateral sclerosis does 
not make a great difference, because in both cases the result is the same: the patient dies. Judged from the consequences, refusing euthanasia does not seem to be an option, because it will leave the patient with the horrible prospect of suffocation. This approach can be endorsed by various consequentialist theories as utilitarianism or pragmatism. Ethical discussion is inhibited here by the fact that the problem is considered to be medical rather than ethical. A good doctor should not deliver his patient to unnecessary suffering.

A third response is made by students who consider ethical discussion to be futile because any ethical point of view eventually refers to one's individual preferences. In this particular case, the fact that so many arguments for and against euthanasia are listed in the medical ethics handbook confirms their conviction that ethical lines of reasoning can sustain and justify any possible viewpoint. Eventually, however, one's stance is determined by personal factors like individual history and education that cannot be analysed or argued further. This approach is supported by emotivist theories that identify ethical stances as personal preferences. Again ethical discussion is avoided and made impossible.

A fourth response that is met in the discussions after watching the video is connected with the religious background of the students. Although the majority of Dutch students explicitly lack a religious orientation, some medical students are very strongly religious. The fact that their religious community forbids euthanasia is considered to be a strong argument. Students who consider themselves to be non-religious see it as their moral duty to respect another person's religious orientation. Here the individual liberalism that promotes the respect of non-religious students strikes an alliance with deontologism by the common idea that the ethical viewpoints of a religious community are beyond argumentation. Again any ethical discussion is made impossible, or rather suffocated under the veil of tolerance.

\section{RELEVANT FACTORS BEYOND ETHICS}

The fact that all responses can be endorsed by ethical theories can be-and actually is-interpreted as a signal that ethics does not amount to anything. Apart from this problematic situation intrinsic to ethics-a subject that will be addressed below-there are a number of relevant factors outside moral theory that make the relation between medicine and ethics a problematic one.

The first factor concerns the nature of medicine as an art. Medicine is a practical science aimed at intervention in order to promote or restore health. That means that medicine, as an art, is result oriented by nature. Whereas philosophy is interested in defining problems rather than solving them, medicine has an entirely different attitude towards problems. The very nature of medicine as result oriented-often being forced to take decisions within a short time span-has the effect that consequentialist theories accord with medicine very well. ${ }^{7}$

The second factor concerns the curriculum of medicine, the place of ethics in it and the influence of other disciplines on ethics. In the curriculum ethics is only one of the disciplines surrounding the core business of becoming a good doctor. Moreover, within the curriculum, courses like medical decision making have an explicitly result oriented character. Students, who are trained in approaching problems from such a perspective, are likely to adopt a pragmatist or consequentialist stance in ethics. All this has been described as the influence of the so called "hidden curriculum": the process of socialisation by which students learn how to "cease" to be a lay person. ${ }^{8}$ This hidden curriculum is taught in many ways - for example, the effect of role models, their stories, jokes, anecdotes, and the messages about their science. ${ }^{9}$
The third factor concerns the context of medicine, being part of contemporary culture. As North Atlantic culture is dominated by liberal individualism, the so called ethical questions are no longer primarily seen in their social dimension. Ethical decisions are delegated to the personal life sphere; a sphere that is very much individualised.

The fourth and last factor concerns the average age of medical students. As the ancient Greek philosopher Aristotle already noticed, studying ethics is more likely to succeed when students have reached the maturity of a certain age. Maturity helps seeing the tragic and complex nature of reality. Apart from Aristotle's argument, there are other reasons why medical students can be prone to not giving priority to ethics. Students rarely choose to study ethics, and are often not concentrating in the obligatory ethics classes. Another reason is the fact that at a relatively young age medical students have to cross a number of borders that are considered a taboo in society, like dealing with corpses. As a coping mechanism detachment is a natural reaction. ${ }^{10}$

\section{ADDRESSING EVASION}

If the above said is true, teaching medical ethics is a tough enterprise as it is hampered by many factors inside ethics, medicine, and culture. On the other hand, however, it is clear that the seriousness of the situation depends on the ethical perspective one adopts. The devoted liberal individualist discussed above will judge the situation as less problematic than those who advocate communitarianist ethics. What does this mean?

In the first place, this means that ethics cannot claim the same neutral status as mathematics. Depending on the ethical theory one (implicitly) adheres, certain tendencies in society are endorsed and promoted. Methodically one reaches a complex problem here in education, for it implies that ethical education asks for both learning to work with conceptual tools and learning to see the social-and, in the end, political-implications of working with one tool rather than another.

In the second place, it means that some ethical theories tend to undermine their own enterprise: working from a strictly consequentialist approach, for example, means to eventually replace ethics by medical decision making.

As a result of this, I see a number of requirements that should be met lest ethical education in medical science is unsuccessful. In the first place, the elements of ethical evasion should be recognised. In the second place, an ethical theory should be promoted that covers all elements that seek for evasion. At this point I will have to put my cards on the table and specify my own account of ethics.

In my view a good ethical theory should help understanding the huge importance of ethics as a human activity that sustains the quality (or the good) of individual and social (or political) life. In order to do so, the cognitive and evaluative nature of emotions should be worked out, otherwise doing ethics will either be an intellectual and emotionally sterile mind game of no practical interest, or-in its subjectivist version-a form of cultivated irrationality. The question, however, is how to make this connection between emotions and ethics.

In agreement with Paul Ricoeur, I propose to define the ethical intention as "aiming at the good life, with and for others in just institutions". ${ }^{11}$ This definition joins two important ethical traditions. The first half of the definition points back to the Aristotelian tradition; in the second half the heritage of Immanuel Kant is honoured.

From the perspective of ethics the emotional response of students can be connected to both traditions, because in both traditions emotions play a role. In the tradition of Aristotle emotions appear as "raw material" that ask to be cultivated 
and developed into virtues. In the Kantian tradition the ethical intention implies the commitment to an objective that is inherently social by nature: the good life with and for others. The motivation for this commitment-however rational Kant's conception of autonomy may seem at first sight-is dependent on the good will. The willingness to be rational and moral implies an attitude of empathy.

The connection between emotions and ethics would be even stronger and more convincing if emotions could be shown to have ethical implications by their very nature as human reactions. Moreover, within the context of teaching medical students discussed above, one would have a good point of departure for addressing the evasion, for all students have emotions with regard to ethical questions. As the history of philosophy shows, the relation between emotions and ethics is a rather complex one. ${ }^{12}$ One would need a good philosophical emotion theory to build such a bridge. In my view, Martha Nussbaum, in her book Upheavals of thought, offers such a philosophical theory. ${ }^{3}$

\section{THE MORAL CONTENT OF EMOTIONS}

Martha Nussbaum develops her emotion theory on ancient Stoic foundations and in discussion with a number of contemporary psychological theories. Contrary to theories that consider emotions to be irrational forces, she convincingly makes clear that emotions always imply an object, are intentional by nature, embody certain beliefs about the object, and are concerned with values. In her own words, emotions "involve judgments about important things, judgments in which, appraising an external object as salient for our own wellbeing, we acknowledge our own neediness and incompleteness before parts of the world that we do not fully control."13 For this reason emotions eventually tell about a person's flourishing, and are, as such, "eudaimonistic" (in the ancient Greek sense, hence the spelling) of nature.

Ethics concerns the realm of freedom. Although dealing with emotions is a necessary element of ethical deliberation, rationality brings in this freedom by enabling critical examination of one's emotional response. Emotions may be focusing on objects that are harmful to oneself, one's neighbour, or wider society. They may undermine the good life, with and for others in just institutions, and it may therefore be important to redirect one's attention. Thus, one can discover that, for example, a rather poor emotional response to the video discussed above points to an ethical stance of individualistic indifference that is hard to defend when one reflects about the ethical consequences of this position.

The cognitive character of Nussbaum's emotion theory does not entail that emotions are always easy to interpret. In discussion with a number of psychological theories, she works out the multilayered texture of emotions-hence the title of the book, referring to a passage of Proust's Remembrance of things past in which emotions are compared with geological upheavals that shape the landscape of our mental and social lives. ${ }^{3}$

Although Nussbaum's theory has the pretension of being a philosophical account that is open to use in different ethical theories, it makes clear from the outset that emotions are not morally neutral entities. People reveal what and who they are by the things that move them. In this way, emotions always reflect a specific cultural context in which particular things are viewed as valuable or good.

Does this mean that emotions are completely determined by culture? Nussbaum develops an account of the cultural influence between two extreme camps: the theorists who completely ignore the role of society on the one hand and the social constructionists that are blind to the variations of individuals on the other. ${ }^{14}$ She provides a matrix for under- standing the sources of intersocietal differences in the emotional life: physical conditions, metaphysical beliefs, practices, language, and social norms. Next to these sources, the social variation of emotions is established by differences in the criteria for their appropriate behavioural manifestation, judgments about the worth of an entire emotion category, views about the appropriate objects for an emotion, and emotion taxonomies.

It is important to see that no matter what the exact interaction between particular individual and cultural context looks like, emotions remain an utmost personal category. They are so personal that they can sometimes surprise one. Thus understanding one's own emotional reaction contributes to understanding oneself.

I think Nussbaum is right in her view that emotions are a very important point of departure for ethical reflection and education-they need to be interpreted, understood, and cultivated in the form of virtue ethics. This is precisely what makes us human and distinguishes us from the emotional life we share with all higher mammals.

\section{AVOIDING EVASION}

Having reached this point how could Nussbaum's theory of emotions help in avoiding the four kinds of evasion discussed above? I shall first explore how Nussbaum's theory faces the evasions on a theoretical level, and then offer some suggestions as to how the evasion could be dealt with in practice.

To the first attitude of evasion-the popular version of liberal individualism—Nussbaum's theory would point out that in its most extreme form this theory is based on a conception of man that is untenable, being opposed to modern insights into the development and flourishing of human beings. Human beings are corporeal, emotional, and relational beings from the very beginning of their existence, and therefore they are marked by dependency and fragility. Those who deny this for the sake of independence and individualism betray their own roots.

The second evasion-consequentialism-is questioned by the fact that apparently ethical questions are more than matters of calculation. The fact that so many of the students are emotionally involved in the video on euthanasia shows that there is more to determining the human good than just the outcome or consequences of human actions. Apparently emotions play a role, which makes aware of the fact that human beings are what Charles Taylor calls "self-interpreting animals" who live by meaning and interpretation. ${ }^{15}$ The symbolic dimension of actions and words impose themselves, especially at important moments in life.

To the third evasion-emotivism-Nussbaum's theory of emotion shows how emotions are always more than just subjective preferences. By showing what is important to people, they reveal a hierarchy of values and they are related to the confirmation or rejection of the (political) state of affairs that embody these values.

To the evasion by the deontology of respect, Nussbaum's theory could point to the limits of this attitude. When it comes to the circumcision of young women, for example, only a few Western students would be able to maintain their attitude of respect. Most of them would react with indignition and it would be easy to show the implicit political content of this emotional response with the help of the emotion theory developed in Upheavals of thought. ${ }^{3}$

\section{PRACTICAL RECOMMENDATIONS}

As a conclusion to these considerations, let us return to the case discussed and end with some practical recommendations that show how the evasion can be avoided by addressing emotions on the practical level of teaching ethics. 
After having shown the video, and having given the opportunity to share some spontaneous reactions, one might continue with a discussion in three steps. The first step is to methodically subtract all relevant medical, legal, and practical perspectives, until one reaches the ethical point of view, in order to show that ethics cannot be reduced to other sciences. Once students see that the nature of a moral problem lies in a conflict between values or principles (in the case of euthanasia, the respect for life versus the relief of suffering), it is important to show how both values or principles are connected to one's emotional response. At this stage, however, there are still many ways of evading ethics by skepticism, relativism, or emotivism.

The next step would be to see and identify the implicit moral content of one's emotions. In this case one can ask questions like "what does it mean when one is touched by the video that shows a man being put to death? What does one's emotional response tell about one's own fears or desires? How is the video interpreted? What anthropological presuppositions come to the fore when one's reaction is analysed?" In the case of an ethical problem to which the students are (emotionally) indifferent-which is not infrequently-one may wonder why the students do not manage to relate to this problem.

Subsequently, the third step might be to clarify the nature of the moral point of view, containing both a moment of personal emotional commitment ("aiming at the good life") and a more formal and social engagement ("with and for others in just institutions"). Within this framework a discussion may be held in which both dimensions are explored. One could begin asking as to whether the students can identify with the physician in the video. This may be the beginning of a discussion about the professional role of the doctor and the consequences for society. In this way there is a close connection established between the motivation of the student to become a physician and the questions that concern our "aiming at the good life, with and for others in just institutions". ${ }^{16}$ Thus the four manners of evasion of ethical discussion are avoided, because both the student's commitment and the inherently social dimension of ethics are secured.

All this does not mean that the present stress on literature and attitude is less relevant. To the contrary: both are very useful ways of training and cultivating moral sensitivity. When done, however, on the basis of a philosophical theory of emotions, a solid foundation is offered that makes sure that education in medical ethics addresses the best of the students' minds and hearts.

\section{REFERENCES}

1 Perkins HR, Geppert CMA, Hazuda HP. Challenges in teaching ethics in medical schools. Am J Med Sci 2000;319:273-8.

2 Coles R. The moral education of medical students. Acad Med 1998;73:55-58.

3 Nussbaum MC. Upheavals of thought. The intelligence of emotions. Cambridge: Cambridge University Press, 2001

4 Ikon Television Network, Death on request, 1994.

5 Hendin H. Selling death and dignity. Hastings Cent Rep 1995;25:19-23.

6 Ten Have HAMJ, Ter Meulen RHJ, Van Leeuwen E. Medische ethiek. Houten: Bohn Stafleu van Loghum, 1998:347-77.

7 See reference 1:274.

8 Hafferty F, Fanks R. The hidden curriculum, ethics teaching and the structure of medical education. Acad Med 1994;69:861-71.

9 Paice E, Heard S, Moss F. How important are role models in making good doctors? BMJ 2002;325:707-10.

10 Finlay S, Fawzy M. Becoming a doctor. J Med Ethics: Medical Humanities $2001 ; 27: 90-2$.

11 Ricoeur P. Oneself as another. Chicago and London: University of Chicago Press, 1992:172.

12 De Sousa R. The rationality of emotion. Cambridge, MA: MIT Press, 1987.

13 See reference 3:19.

14 See reference 3:139-173.

15 Taylor C. Human agency and language. Philosophical papers I. Cambridge, UK: Cambridge University Press, 1985:45.

16 See reference 11:172. 